\title{
On Non-linearities Separate Modeling Method and State Feedback Control
}

\author{
Han Zhongxu ${ }^{1, a}$, Tian Xinshou ${ }^{1,2, b}$ \\ 1 China Electric Power Research Institute, Haidian District, Beijing 100192, China \\ 2 North China Electric Power University, Changping District, Beijing 102206, China \\ a zhongxuh@epri.sgcc.com.cn, ${ }^{b}$ tianxinshou@epri.sgcc.com.cn
}

\begin{abstract}
Keywords: modeling method; non-linearities; increment function observer (IFO-K $\triangle \mathrm{x}$ ); state feedback; slow-time-varying; Sylvester matrix equation.
\end{abstract}

Abstract. Modeling method of non-linearities objects controlled have more problem and difficulty. Based on the increment function observer (IFO-K $\triangle \mathrm{x}$ ), this paper gives the mathematical conceptions of Increment Function Observer with variable gain IFO-K(T(t)) $\triangle \mathrm{x}$ and the Increment full dimension State Observer with variable gain ISO- $T(t) \triangle x$, which makes the application of increment observer extending to non-linear slow-time-varying controlled system. In this paper, the sufficient condition of IFO-K(T $(\mathrm{t})) \triangle \mathrm{x}$ and ISO- $\mathrm{T}(\mathrm{t}) \triangle \mathrm{x}$ is presented, and comparing this condition with the sufficient and necessary condition of increment function observer, the useful conclusion for practical engineering can be obtained that the design of increment observer can avoid seeking the solution of Sylvester matrix equation, which provides the more maturity theory foundation for the engineering application of incremental observer.

\section{Introduction}

Many relevant literatures ${ }^{[1-7]}$ in the field of pyrology have pointed out that the controlled object of unit generator coordinated control system is a multivariable systems with non-linear, slow-time-varying parameters, hysteresis and large inertia, and the hysteresis and inertia changes with long-term operating of the boiler equipment or changing of load. It is difficult to design a controller or an observer using the traditional research method, because the matrix $\boldsymbol{A} 、 \boldsymbol{B} 、 \boldsymbol{C}$ of the controlled object is considered to be known in linear system theory. It is sometimes change that state space description $\boldsymbol{A} 、 \boldsymbol{B} 、 \boldsymbol{C}$ and transfer function description $\mathrm{G}(s)=\boldsymbol{C}(s \boldsymbol{I}-\boldsymbol{A})^{-1} \boldsymbol{B}$ of controlled object in the practical project, and peak-valley difference of the current grid load demand become bigger, so it is inevitable large capacity units involved in peaking, thereby the control of the unit must be able to adapt to the substantial changes of load. The non-linearity and slow-time-varying in the dynamic process of controlled object is show, which have a great effect to the operation performance of the control system, due to the changes of operating point in unit power plant.

The research work ${ }^{[8,9]}$ on increment function observer IFO- $\boldsymbol{K} \Delta \boldsymbol{x}$ provides a method on how to design non-linear controlled systems observer, and there are some actual results ${ }^{[10-15]}$ in practical engineering. Considering slow-time-varying of controlled objects in stability robustness of the observer $^{[16]}$ doesn't affect stability of IFO- $\boldsymbol{K} \Delta \boldsymbol{x}$ through observational facts, but it is lack of theoretical analysis and argumentation.

The research work ${ }^{[9]}$ proved non-linear system based on Hammerstein model ${ }^{[17]}$ after the concept of the IFO- $\boldsymbol{K} \Delta \boldsymbol{x}$ was proposed in paper[8]. When designing feedback control system using increment function observer, the controller and observer can be designed independently that separation principle $^{[18]}$ is applied to non-linear systems based on Hammerstein model. The pole is made up of closed-loop system pole $\sigma\left[\boldsymbol{A}-\boldsymbol{B} \boldsymbol{f}^{\prime}(\xi) \boldsymbol{K}\right]$ under state feedback law and pole $\sigma(\boldsymbol{F})$ of increment function observer when certain conditions are met. The necessary and sufficient conditions of IFO- $K \Delta \boldsymbol{x}$ is studied further in paper[19], which is different with The sufficient conditions in paper[8], there is no $\boldsymbol{H}=\boldsymbol{T} \boldsymbol{B}$, which is the very important difference between IFO- $\boldsymbol{K} \Delta \boldsymbol{x}$ and Luenberger function observer. 
A worth exploring ways is provided that whether it can be avoided seeking the solution of Sylvester matrix equation in the designing process of IFO- $\boldsymbol{K} \Delta \boldsymbol{x}$ in theoretical research.

The paper looks at the practical control engineering, and the perturbations affect of unmodeled characteristics, non-linear and slow-time-varying to controlled object is fully considered. In order to make it more in line with the actual characteristics of the unit generator coordinated controlled object, the matrix $\boldsymbol{F}, \boldsymbol{G}$ and $\boldsymbol{M}$ in observer is considered to be known, and the state space description of the controlled system: matrix $\boldsymbol{A}(t), \boldsymbol{B}(t), \boldsymbol{C}(t)$ is considered variable parameters. What changes will occur on incremental function observer, what is it that the necessary and sufficient conditions of solution matrix T existed on Sylvester matrix equation (18)? Whether it can be avoided seeking the solution of Sylvester matrix equation in the designing process of IFO- $\boldsymbol{K} \Delta \boldsymbol{x}$ ?

\section{Mathematical Description of Problem- a Non-linear Separate Mode Based on Hammerstein Model}

The non-linear system based on Hammerstein model can be represented by linear controlled system and a group high order polynomial.

$$
\left\{\begin{array}{l}
\dot{x}=A x+B u, \quad x\left(t_{0}\right)=x_{0} \\
y=C x \\
u=f(v)
\end{array}\right.
$$

Among, $\boldsymbol{x} \in \boldsymbol{R}^{n} ; \boldsymbol{A} 、 \boldsymbol{B} 、 \boldsymbol{C}$ are proper order real matrix, $\boldsymbol{v} 、 \boldsymbol{y} 、 \boldsymbol{u}$ are proper dimensional input and output vector, $\mathrm{f}$ is proper dimensional non-linear functional relationship.

In order to make studied object have a broader significance, the following expand is given for above Hammerstein model to describe non-linear slow-time-varying n- dimensional controlled system. So there have the following definitions: the non-linear system based on slow time-variant Hammerstein model can be represented by linear slow-time-varying controlled system and a non-linear gain group high order polynomial.

$$
\begin{aligned}
& \left(\begin{array}{l}
\dot{\boldsymbol{x}}=\boldsymbol{A}(t) \boldsymbol{x}+\boldsymbol{B}(t) \boldsymbol{u}, \quad \boldsymbol{x}\left(t_{0}\right)=\boldsymbol{x}_{\boldsymbol{v}} \\
\boldsymbol{y}=\boldsymbol{C}(t) \boldsymbol{x}
\end{array}\right. \\
& \boldsymbol{u}=\boldsymbol{f}(\boldsymbol{v}) \\
& \boldsymbol{x} \in \boldsymbol{R}^{n} ; \boldsymbol{A}(t) 、 \boldsymbol{B}(t) 、 \boldsymbol{C}(t) \text { are proper order time-variant real matrix. }
\end{aligned}
$$

\section{Transfer Function Description and State Space Description of Linear System Transformation}

Paper[20] detailed describe the Interconversion processing between transfer function description and state space description.

When all poles of transfer function are not the same, the part fractional of the transfer function is described as follows:

$$
W(s)=b_{o}+\frac{r_{1}}{s+s_{1}}+\frac{r_{2}}{s+s_{2}}+\cdots+\frac{r_{k}}{s+s_{k}}
$$

The matrix form as follows:

$$
\left[\begin{array}{c}
\dot{\boldsymbol{x}}_{1} \\
\dot{\boldsymbol{x}}_{2} \\
\vdots \\
\dot{\boldsymbol{x}}_{k}
\end{array}\right]=\left[\begin{array}{cccc}
-s_{1} & 0 & \cdots & 0 \\
0 & -\mathrm{s}_{2} & \ddots & \vdots \\
\vdots & \ddots & \ddots & 0 \\
0 & \cdots & 0 & -s_{k}
\end{array}\right]\left[\begin{array}{c}
\boldsymbol{x}_{1} \\
\boldsymbol{x}_{2} \\
\vdots \\
\boldsymbol{x}_{k}
\end{array}\right]+\left[\begin{array}{c}
1 \\
1 \\
\vdots \\
1
\end{array}\right] u \quad \quad \boldsymbol{y}=\left[\begin{array}{llll}
r_{1} & r_{2} & \cdots & r_{k}
\end{array}\right]\left[\begin{array}{c}
\boldsymbol{x}_{1} \\
\boldsymbol{x}_{2} \\
\vdots \\
\boldsymbol{x}_{k}
\end{array}\right]+\left[b_{o}\right] u
$$

When the poles of transfer function is a pair of conjugate complex roots, the part fractional of the transfer function is described as follows:

$$
W_{1,2}(s)=\frac{\kappa+j \gamma}{s+\sigma+j \omega}+\frac{\kappa-j \gamma}{s+\sigma-j \omega}=\frac{2 \cdot[\kappa s+(\kappa \sigma-\omega \gamma)]}{s^{2}+2 \sigma \cdot s+\sigma^{2}+\omega^{2}}
$$

Then, the matrix form as follows: 


$$
\left[\begin{array}{l}
\dot{\boldsymbol{x}}_{1} \\
\dot{\tilde{\boldsymbol{x}}}_{2}
\end{array}\right]=\left[\begin{array}{cc}
0 & 1 \\
-\left(\sigma^{2}+\omega^{2}\right) & -2 \sigma
\end{array}\right]\left[\begin{array}{l}
\tilde{\boldsymbol{x}}_{1} \\
\tilde{\boldsymbol{x}}_{2}
\end{array}\right]+\left[\begin{array}{l}
0 \\
1
\end{array}\right] u \quad \boldsymbol{y}_{1,2}=[2 \cdot(\kappa \sigma-\omega \gamma) \quad 2 \kappa]\left[\begin{array}{c}
\widetilde{\boldsymbol{x}}_{1} \\
\widetilde{\boldsymbol{x}}_{2}
\end{array}\right]
$$

When the poles of transfer function are equal, the part fractional of the transfer function is described as follows:

$$
W_{i}(s)=\frac{r_{1 i}}{s+s_{i}}+\frac{r_{2 i}}{\left(s+s_{i}\right)^{2}}+\cdots+\frac{r_{k i}}{\left(s+s_{i}\right)^{k_{i}}}
$$

Then, the matrix form as follows:

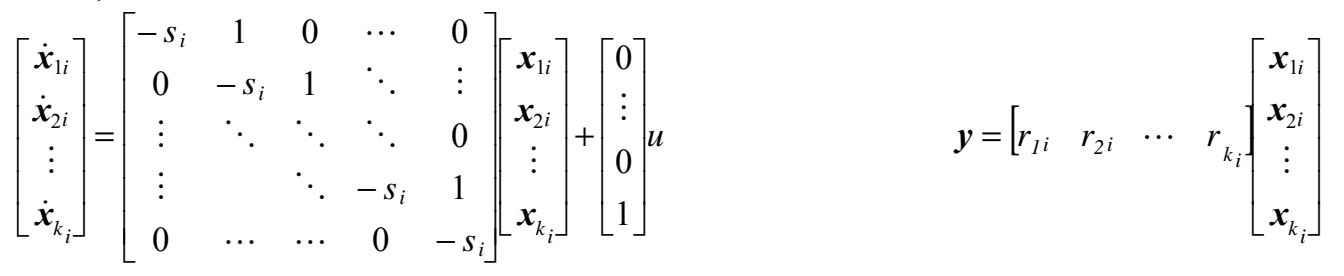

When the transfer function is described by rational fraction

$$
G(s)=\frac{b_{m} s^{m}+b_{m-1} s^{m-1}+\cdots+b_{1} s+b_{0}}{s^{n}+a_{n-1} s^{n-1}+\cdots+a_{1} s+a_{0}}
$$

If $m \leq n$, the $G(s)$ is real rational fraction on $\mathrm{s}$, and the matrix form as follows:

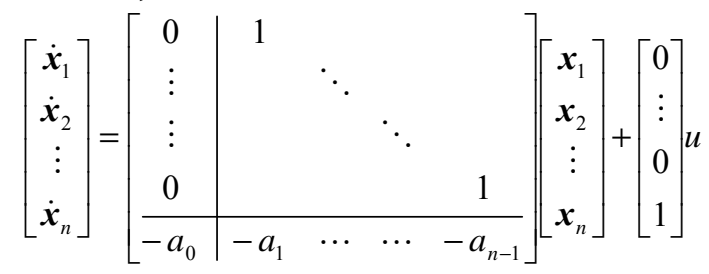

$$
\boldsymbol{y}=\left[\begin{array}{llllll}
b_{0} & \cdots & b_{m} & 0 & \cdots & 0
\end{array}\right]\left[\begin{array}{c}
\boldsymbol{x}_{1} \\
\boldsymbol{x}_{2} \\
\vdots \\
\boldsymbol{x}_{n}
\end{array}\right]
$$

If $m=n$, the matrix form as follows:

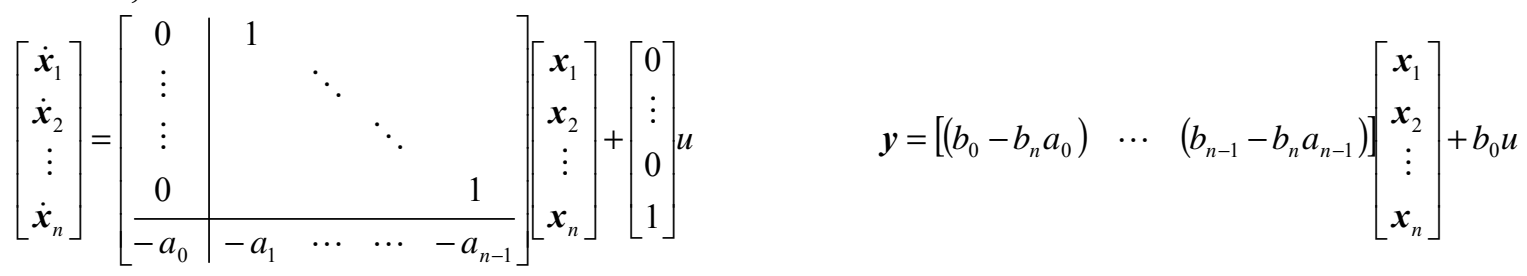

The research has proved that a Increment Function Observer IFO- $\boldsymbol{K} \Delta \boldsymbol{x}$ is composed of the following observability system and simultaneous system(1)-(2), if the simultaneous system(1)-(2) is observability and matrix $\boldsymbol{K} \in \boldsymbol{R}^{r \times n}$ is known.

$$
\left\{\begin{array}{l}
\dot{z}=\boldsymbol{F} z+\boldsymbol{G} \Delta \hat{\boldsymbol{y}}+\boldsymbol{H} \Delta \hat{\boldsymbol{u}}, \quad z\left(t_{0}\right)=z_{0} \\
\boldsymbol{w}=\boldsymbol{M z}+\boldsymbol{N} \Delta \hat{\boldsymbol{y}} \\
\Delta \hat{\boldsymbol{u}}=\boldsymbol{v}-\boldsymbol{v}_{\boldsymbol{D} M \boldsymbol{D}} \\
\boldsymbol{v}_{\boldsymbol{D M D}}=\boldsymbol{f}_{v}\left(R_{D M D}\right) \\
\Delta \hat{\boldsymbol{y}}=\boldsymbol{y}-\boldsymbol{y}_{\boldsymbol{D} M D} \\
\boldsymbol{y}_{D M D}=\boldsymbol{f}_{\boldsymbol{y}}\left(R_{D M D}\right)
\end{array}\right.
$$

Where $\boldsymbol{z} \in \boldsymbol{R}^{p} ; \boldsymbol{F}, \boldsymbol{H}, \boldsymbol{G}, \boldsymbol{N}, \boldsymbol{M}$ are proper order real matrix, $\boldsymbol{w} 、 \boldsymbol{v}_{\boldsymbol{D M D}}, \boldsymbol{y}_{\boldsymbol{D} M \boldsymbol{D}}, \boldsymbol{R}_{\boldsymbol{D M D}} \Delta \hat{\boldsymbol{u}} 、 \Delta \hat{\boldsymbol{y}}$ are proper dimensional input and output vector, $\boldsymbol{f}_{\boldsymbol{v}}$ and $\boldsymbol{f}_{\boldsymbol{y}}$ are proper dimensional non-linear functional relationship, $\boldsymbol{R}_{\boldsymbol{D M D}}$ can be obtained by measuring, and $\left[\begin{array}{ll}\boldsymbol{F} & \boldsymbol{M}\end{array}\right]$ is observability. The necessary and sufficient conditions of IFO- $\boldsymbol{K} \Delta \boldsymbol{x}^{[19]}$ made up of simultaneous system(1)-(2) as follows:

(1) $\operatorname{Re} \lambda(\boldsymbol{F})<0$

(2) $v_{D M D}=f_{v}\left(R_{D M D}\right)= \begin{cases}\boldsymbol{v}-\frac{d \boldsymbol{f}(\boldsymbol{v})}{\boldsymbol{d} \boldsymbol{v}} \Delta \boldsymbol{v} & t \in\left[t_{0},\right. \\ \boldsymbol{v} & t \in\left[t_{1}, \infty\right]\end{cases}$

(3) $\boldsymbol{y}_{D M D}=\boldsymbol{f}_{\boldsymbol{y}}\left(R_{\boldsymbol{D} M D}\right)=\boldsymbol{C} \boldsymbol{x}_{\boldsymbol{0}}$

The proper order real matrix $T$ is existence, which meet the following formula:

(4) $\boldsymbol{T A}-\boldsymbol{F T}=\boldsymbol{G C}$ 
(5) $M T+N C=K$

Obviously, compared with the following sufficient condition ${ }^{[8]}$ of IFO- $\boldsymbol{K} \Delta \boldsymbol{x}$ :

(1) $\operatorname{Re} \lambda(\boldsymbol{F})<0$

And the proper order real matrix $\boldsymbol{T}$ is existence, which meet the following formula:

(2) $\boldsymbol{H}=\boldsymbol{T} \boldsymbol{B}$

(3) $\boldsymbol{T A}-\boldsymbol{F T}=\boldsymbol{G C}$

(4) $M T+N C=K$

(5) $\Delta \hat{\boldsymbol{u}}=\Delta \boldsymbol{u}$

(6) $\Delta \hat{\boldsymbol{y}}=\Delta \boldsymbol{y}$

The necessary and sufficient conditions of IFO- $\boldsymbol{K} \Delta \boldsymbol{x}^{[19]}$ have practical guidance for practical engineering.

The matrix $\mathrm{F}$ is stability that $\operatorname{Re} \lambda(\boldsymbol{F})<0$ usually can be done for an actual engineering problem, because $\mathrm{F}$ is parameter of observer. The matrix $\mathrm{F}$ is concerned with the controlled object in the different extent in the other conditions, in particular $\boldsymbol{T A}-\boldsymbol{F T}=\boldsymbol{G C}$, and the Sylvester matrix equation should be solved. Even a function observer has be obtained, there is also an actual problem that whether the observer can also work well when a parameter of the controlled object change, without considering the problem and trouble in the solving process.

Unlike previous research methods, the attention of IFO- $\boldsymbol{K} \Delta \boldsymbol{x}$ Does not lie in solving the Sylvester matrix equation, but designing an increment function observer based on qualitative understanding of the controlled system, and then examining its robustness. The focus of the idea on robustness research of Sylvester matrix equation, he problem boils down to:

What is it that the necessary and sufficient conditions of solution matrix T existed on Sylvester matrix equation (18)?

The solution matrix T on Sylvester matrix equation (18) is related with what factors?

Is it possible to avoid solving Sylvester matrix equation in the processing of designing increment function observer IFO- $\boldsymbol{K} \Delta \boldsymbol{x}$ ?

If the studied object extends to non-linear slow-time-varying Hammerstein system based on simultaneous system(1)-(2), what aspects of increment function observer IFO- $\boldsymbol{K} \Delta \boldsymbol{x}$ need to be improved, and whether related answers and conclusions can be given to the above question.

\section{Mathematical Analysis}

\section{Lemma.}

《linear system theory》 ${ }^{[21]}$ proves that the necessary conditions of nonsingular $n \times n$ solution matrix T existed on Sylvester matrix equation $\boldsymbol{T A}-\boldsymbol{F T}=\boldsymbol{G C}$, which is helpful to problems.

Lemma1: [the necessary conditions that solution matrix $\mathrm{T}$ on Sylvester matrix equation of increment full dimension state observer with multi-output is nonsingular]Taking the multiple-output n-dimensional continuous linear time invariant observing system (1) and the following full-dimensional state observer for research object.

$$
\left\{\begin{array}{l}
\dot{z}=\boldsymbol{F} \boldsymbol{z}+\boldsymbol{G} \boldsymbol{y}+\boldsymbol{H u}, \quad \boldsymbol{z}\left(t_{\boldsymbol{o}}\right)=\boldsymbol{z}_{0} \\
\hat{\boldsymbol{x}}=\boldsymbol{T}^{-1} \boldsymbol{z}
\end{array}\right.
$$

If all eigenvalues of the matrix $\boldsymbol{F} \in \boldsymbol{R}^{n \times n}$ have a negative real part, and there is no public eigenvalue with matrix $\boldsymbol{A}$, then the necessary conditions of nonsingular $n \times n$ solution matrix $\mathrm{T}$ existed on Sylvester matrix equation $\boldsymbol{T A}-\boldsymbol{F T}=\boldsymbol{G C}$ is that $\{\boldsymbol{A}, \boldsymbol{C}\}$ are complete observability, and $\{\boldsymbol{F}, \boldsymbol{G}\}$ is complete controllable.

Lemma2: [the necessary and sufficient conditions that solution matrix $\mathrm{T}$ on Sylvester matrix equation of increment full dimension state observer with single output is nonsingular] Taking the single output $\mathrm{n}$-dimensional continuous linear time invariant observing system (1) and the full-dimensional state observer(26) for research object. If all eigenvalues of the matrix $\boldsymbol{F} \in \boldsymbol{R}^{n \times n}$ have a negative real part, and there is no public eigenvalue with matrix $\boldsymbol{A}$, then the necessary and sufficient 
conditions of nonsingular $n \times n$ solution matrix T existed on Sylvester matrix equation $\boldsymbol{T A}-\boldsymbol{F T}=\boldsymbol{G C}$ is that $\{\boldsymbol{A}, \boldsymbol{C}\}$ are complete observability, and $\{\boldsymbol{F}, \boldsymbol{G}\}$ is complete controllable.

We can obtain the following Lemma3 through the proof processing of Lemma1.

Lemma3:[A relational equation on solution matrix $T$ on Sylvester matrix equation of increment full dimension state observer with multi-output] Taking the multiple-output n-dimensional continuous linear time invariant observing system (1) and the full-dimensional state observer (26)for research object. If all eigenvalues of the matrix $\boldsymbol{F} \in \boldsymbol{R}^{n \times n}$ have a negative real part, and there is no public eigenvalue with matrix $\boldsymbol{A}$, then $n \times n$ solution matrix $\mathrm{T}$ of Sylvester matrix equation $\boldsymbol{T A}-\boldsymbol{F T}=\boldsymbol{G C}$ is existence, and the following relational equation is existence(q is dimension of the output vector $\mathrm{y})$ :

$$
\boldsymbol{T}=-[\alpha(\boldsymbol{F})]^{-1} \boldsymbol{U}_{F} \boldsymbol{\Lambda}_{q} \boldsymbol{V}_{\mathrm{A}}
$$

where

$$
\begin{aligned}
\underset{n q \times n q}{\boldsymbol{A}_{q}} & =\left[\begin{array}{ccccc}
\boldsymbol{\alpha}_{1} \boldsymbol{I}_{q} & \alpha_{2} \boldsymbol{I}_{q} & \cdots & \boldsymbol{\alpha}_{n-1} \boldsymbol{I}_{q} & \boldsymbol{I}_{q} \\
\boldsymbol{\alpha}_{2} \boldsymbol{I}_{q} & \alpha_{3} \boldsymbol{I}_{q} & \cdots & \boldsymbol{I}_{q} & 0 \\
\vdots & \vdots & \vdots & \vdots & \vdots \\
\boldsymbol{\alpha}_{n-1} \boldsymbol{I}_{q} & \boldsymbol{I}_{q} & \cdots & 0 & 0 \\
\boldsymbol{I}_{q} & 0 & \cdots & 0 & 0
\end{array}\right] \\
\underset{n \times n q}{\boldsymbol{U}_{F}} & =\left[\begin{array}{llll}
\boldsymbol{G} & \boldsymbol{F G} & \cdots & \boldsymbol{F}^{n-1} \boldsymbol{G}
\end{array}\right]
\end{aligned}
$$

$\underset{n q \times n}{V_{A}}=\left[\begin{array}{c}C \\ C A \\ \vdots \\ C A^{n-1}\end{array}\right]$

in Lemma3

$$
\alpha(s)=\operatorname{det}(s \boldsymbol{I}-\boldsymbol{A})=s^{n}+\alpha_{n-1} s^{n-1}+\cdots+\alpha_{1} s+\alpha_{0}
$$

The above formulas describe characteristic polynomial of matrix $\boldsymbol{A}$, we can obtain $\alpha(\boldsymbol{A})=0$ based on Caley-Hamilton theorem. If $\lambda_{i}$ is an eigenvalue of the stable matrix $\boldsymbol{F}, \alpha\left(\lambda_{i}\right)$ is an eigenvalue of the $\alpha(\boldsymbol{F})$. Because there is no public eigenvalue between $\mathrm{A}$ and $\mathrm{F}$, the following equation is established to all eigenvalues $\lambda_{i}(i=1,2, \cdots, n)$ of matrix $\boldsymbol{F}$ :

$$
\alpha\left(\lambda_{i}\right) \neq 0
$$

And the $\alpha(\boldsymbol{A})=0$ and $\alpha(\boldsymbol{F})$ are nonsingular.

\section{Mathematical Definition of Increment Function Observer with Variable Gain.}

Definition1:The complete observability system (14) is called Increment Function Observer with variable gain of simultaneous system(3)-(4). All the states and outputs of simultaneous system(3)-(4)-(14) meet the following asymptotic relationship for any initial value $\boldsymbol{x}_{\boldsymbol{0}}, \boldsymbol{z}_{0}$ and any admissible control $v(t)$.

$$
\lim _{t \rightarrow \infty}[\boldsymbol{w}(t)-\boldsymbol{K}(\boldsymbol{T}(t)) \Delta \boldsymbol{x}(t)]=0
$$

$\boldsymbol{K}(t) \in \boldsymbol{R}^{r \times n}$ is a function of matrix $\boldsymbol{T}(t)$ where, and $\boldsymbol{T}(t)$ is a solution matrix which can meet Sylvester matrix equation $\boldsymbol{T}(t) \boldsymbol{A}(t)-\boldsymbol{F} \boldsymbol{T}(t)=\boldsymbol{G C}(t)$

Definition2: If matrix $N=0$, the complete observability system (14) is called Increment full dimension State Observer with variable gain of simultaneous system(3)-(4). All the states and outputs of simultaneous system(3)-(4)-(14) meet the following asymptotic relationship for any initial value $x_{0}, z_{0}$ and any admissible control $v(t)$.

$$
\lim _{t \rightarrow \infty}[z(t)-\boldsymbol{T}(t) \Delta \boldsymbol{x}(t)]=0
$$

The matrix $\boldsymbol{T}(t)$ is a nonsingular $n \times n$ solution matrix which can meet Sylvester matrix equation $\boldsymbol{T}(t) \boldsymbol{A}(t)-\boldsymbol{F} \boldsymbol{T}(t)=\boldsymbol{G C}(t)$.

\section{Theorem and Inference.}


Theorem1[ the sufficient conditions of Increment Function Observer with variable gain]:A non-linear system is composed with multiple-output n-dimensional continuous linear slow-time-varying controlled system (3) and a non-linear gain group high order polynomial(4). The simultaneous system(3)-(4) is observability, and matrix $\boldsymbol{M} \in \boldsymbol{R}^{r \times n}$ is known, then the sufficient conditions of IFO- $\boldsymbol{K}(\boldsymbol{T}(t)) \Delta \boldsymbol{x}$ made up of simultaneous system(1)-(2) based on observability system(14) as follows:

(1) $\operatorname{Re} \lambda(\boldsymbol{F})<0$

(2) $v_{D M D}=f_{v}\left(R_{D M D}\right)= \begin{cases}v-\frac{d f(v)}{d v} \Delta v & t \in\left[t_{0}, t_{1}\right) \\ v & t \in\left[t_{1}, \infty\right]\end{cases}$

(3) $y_{D M D}=\boldsymbol{f}_{\boldsymbol{y}}\left(R_{D M D}\right)=\boldsymbol{C}\left(t_{0}\right) \boldsymbol{x}_{\boldsymbol{\theta}}$

(4) $\alpha[\boldsymbol{A}(t)]=0$ and $\alpha(\boldsymbol{F})$ are nonsingular(there is no public eigenvalue between matrix $\boldsymbol{A}(t)$ and $\boldsymbol{F}$ at different times)

(5) $\{\boldsymbol{A}(t), \boldsymbol{C}(t)\}$ is complete observability, and $\{\boldsymbol{F}, \boldsymbol{G}\}$ is complete controllable at any times

(6) $\boldsymbol{M T}(t)+\boldsymbol{N C}(t)=\boldsymbol{K}(t)$

Certificate: assume

$\boldsymbol{\varepsilon}=\boldsymbol{z}-\boldsymbol{T}(t) \Delta \boldsymbol{x}, \quad \boldsymbol{e}=\boldsymbol{w}-\boldsymbol{K}(t) \Delta \boldsymbol{x}$

note:

$\Delta \hat{\boldsymbol{y}}=\boldsymbol{C}(t) \Delta \boldsymbol{x}+\boldsymbol{\delta}_{1} \boldsymbol{I} \cdot \boldsymbol{C}(t) \Delta \boldsymbol{x}, \quad \Delta \hat{\boldsymbol{u}}=\Delta \boldsymbol{u}+\boldsymbol{\delta}_{2} \boldsymbol{I} \cdot \Delta \boldsymbol{u}$

The $\boldsymbol{\delta}_{1}, \boldsymbol{\delta}_{2}$ is vector composed with the unknown Perturbed element, we can get the following relationship from formula (3),(14) and (41) (42).

$\dot{\boldsymbol{\varepsilon}}=\boldsymbol{F} \boldsymbol{\varepsilon}+\left[\boldsymbol{G}\left(\boldsymbol{I}+\boldsymbol{\delta}_{1} \boldsymbol{I}\right) \boldsymbol{C}(t)+\boldsymbol{F} \boldsymbol{T}(t)-\boldsymbol{T}(t) \boldsymbol{A}(t)\right] \Delta \boldsymbol{x}+\left[\boldsymbol{H}\left(\boldsymbol{I}+\boldsymbol{\delta}_{2} \boldsymbol{I}\right)-\boldsymbol{T}(t) \boldsymbol{B}(t)\right] \Delta \boldsymbol{u}$

$\boldsymbol{e}=\boldsymbol{M} \boldsymbol{\varepsilon}+(\boldsymbol{N C}(t)+\boldsymbol{M T}(t)-\boldsymbol{K}(t)) \Delta \boldsymbol{x}+\boldsymbol{N}(\Delta \hat{\boldsymbol{y}}-\Delta \boldsymbol{y})=\boldsymbol{M} \boldsymbol{\varepsilon}+\left[\boldsymbol{N}\left(\boldsymbol{I}+\boldsymbol{\delta}_{1} \boldsymbol{I}\right) \boldsymbol{C}(t)+\boldsymbol{M T}(t)-\boldsymbol{K}(t)\right] \Delta \boldsymbol{x}$

If conditions (2)and(3) in Theorem1 are established, we can get the following relationship in the interval $t \in\left[t_{0}, t_{1}\right)$ according to formula (14) and (42).

$$
\begin{aligned}
\dot{\boldsymbol{\varepsilon}} & =\boldsymbol{F} \boldsymbol{\varepsilon}+[\boldsymbol{G C}(t)+\boldsymbol{F T}(t)-\boldsymbol{T}(t) \boldsymbol{A}(t)] \Delta \boldsymbol{x}+[\boldsymbol{H}-\boldsymbol{T}(t) \boldsymbol{B}(t)] \frac{d \boldsymbol{d}(\boldsymbol{v})}{\boldsymbol{d} \boldsymbol{v}} \Delta \boldsymbol{v} \\
\boldsymbol{e} & =\boldsymbol{M} \boldsymbol{\varepsilon}+[\boldsymbol{N C}(t)+\boldsymbol{M T}(t)-\boldsymbol{K}(t)] \Delta \boldsymbol{x}
\end{aligned}
$$

If conditions (4)and(5) in Theorem1 are established, we can know that the $n \times n$ solution matrix T on Sylvester matrix equation $\boldsymbol{T A}-\boldsymbol{F T}=\boldsymbol{G C}$ is existed as follows formula (27) from Lemmal and Lemma3 according to condition(1) $\operatorname{Re} \lambda(\boldsymbol{F})<0$ in Theorem1, that $\boldsymbol{T}(t)=-[\alpha(\boldsymbol{F})]^{-1} \boldsymbol{U}_{F} \boldsymbol{\Lambda}_{q} \boldsymbol{V}_{A}$. The formula (34) becomes the following relationship:

$$
\begin{aligned}
& \dot{\boldsymbol{\varepsilon}}=\boldsymbol{F} \boldsymbol{\varepsilon}+[\boldsymbol{H}-\boldsymbol{T}(t) \boldsymbol{B}(t)] \frac{\boldsymbol{d f}(\boldsymbol{v})}{\boldsymbol{d} \boldsymbol{v}} \Delta \boldsymbol{v} \\
& \boldsymbol{T}(t)=-[\alpha(\boldsymbol{F})]^{-1} \boldsymbol{U}_{F} \boldsymbol{\Lambda}_{q} \boldsymbol{V}_{A} \text { in condition (6)in Theorem1, we can get that: } \\
& \boldsymbol{e}=\boldsymbol{M} \boldsymbol{\varepsilon}
\end{aligned}
$$

If conditions (1)and(2)in Theorem1 are established, we can get the following relationship in the interval $t \in\left[t_{1}, \infty\right]$.

$$
\lim _{t \rightarrow \infty} \varepsilon(t)=0
$$

Because the formula (3) is a multiple-output $n$-dimensional continuous linear slow-time-varying controlled system, so the parameter of which is different at different times. But we know that formula(38)and(39) are always established in different time periods from conditions (4)and(5) in Theorem1. The $n \times n$ solution matrix T on Sylvester matrix equation is existed, and the matrix T will change because of the change of $\{\boldsymbol{A}(t), \boldsymbol{C}(t)\}$ based on formula (27)-(30), and the feedback matrix $\boldsymbol{K}(t)$ is a function of solution matrix $\mathrm{T}$, which can be described by $\boldsymbol{K}(\boldsymbol{T}(t))$. The following formula is established.

$$
\lim _{t \rightarrow \infty} \boldsymbol{e}(t)=\lim _{t \rightarrow \infty}[\boldsymbol{w}(t)-\boldsymbol{K}(\boldsymbol{T}(t)) \Delta \boldsymbol{x}(t)]=0
$$


Theorem2[ the sufficient conditions of Increment full dimension State Observer with variable gain]:A non-linear system is composed with single-output n-dimensional continuous linear slow-time-varying controlled system (3) and a non-linear gain group high order polynomial(4). The simultaneous system(3)-(4) is observability, and matrix $\boldsymbol{M} \in \boldsymbol{R}^{r \times n}$ is known. When matrix $\boldsymbol{N}=0$ of observability system (14), the sufficient conditions of ISO- $\boldsymbol{T}(t) \Delta \boldsymbol{x}$ made up of simultaneous system(3)-(4) as follows:

(1) $\operatorname{Re} \lambda(\boldsymbol{F})<0$

(2) $v_{D M D}=f_{v}\left(R_{D M D}\right)= \begin{cases}v-\frac{d f(v)}{d v} \Delta v & t \in\left[t_{0}, t_{1}\right) \\ v & t \in\left[t_{1}, \infty\right]\end{cases}$

(3) $y_{D M D}=f_{y}\left(R_{D M D}\right)=C\left(t_{0}\right) x_{0}$

(4) $\alpha[\boldsymbol{A}(t)]=0 \quad \alpha[\boldsymbol{A}(t)]=0$ and $\alpha(\boldsymbol{F})$ are nonsingular(there is no public eigenvalue between matrix $\boldsymbol{A}(t) \quad$ and $\boldsymbol{F} \quad$ at different

(5) $\{\boldsymbol{A}(t), \boldsymbol{C}(t)\}$ is complete observability, and $\{\boldsymbol{F}, \boldsymbol{G}\}$ is complete controllable at any times

(6) $\boldsymbol{M T}(t)=\boldsymbol{K}(t)$

Certificate: If conditions (2) and (3) in Theorem2 are established, we can get the following relationship in the interval $t \in\left[t_{0}, t_{1}\right)$ according to formula(41)and(42), and matrix $N=0$ of observability system (14) in Definition2.

$$
\begin{aligned}
& \dot{\boldsymbol{\varepsilon}}=\boldsymbol{F} \boldsymbol{\varepsilon}+[\boldsymbol{G C}(t)+\boldsymbol{F T}(t)-\boldsymbol{T}(t) \boldsymbol{A}(t)] \Delta \boldsymbol{x}+[\boldsymbol{H}-\boldsymbol{T}(t) \boldsymbol{B}(t)] \frac{d \boldsymbol{f}(\boldsymbol{v})}{\boldsymbol{d} \boldsymbol{v}} \Delta \boldsymbol{v} \\
& \boldsymbol{e}=\boldsymbol{M} \boldsymbol{\varepsilon}+[\boldsymbol{M T}(t)-\boldsymbol{K}(t)] \Delta \boldsymbol{x}
\end{aligned}
$$

If conditions (4)and(5) in Theorem 2 are established, we can know that the $n \times n$ solution matrix $\mathrm{T}$ on Sylvester matrix equation $\boldsymbol{T}(t) \boldsymbol{A}(t)-\boldsymbol{F T}(t)=\boldsymbol{G C}(t)$ is existed as follows formula (27) from Lemma2 and Lemma3 according to condition (1) $\operatorname{Re} \lambda(\boldsymbol{F})<0$ in Theorem2, that $\boldsymbol{T}(t)=-[\alpha(\boldsymbol{F})]^{-1} \boldsymbol{U}_{F} \boldsymbol{\Lambda}_{q} \boldsymbol{V}_{A}$. The formula (57) becomes the following relationship:

$$
\dot{\varepsilon}=\boldsymbol{F} \boldsymbol{\varepsilon}+[\boldsymbol{H}-\boldsymbol{T}(t) B(t)] \frac{d \boldsymbol{f}(\boldsymbol{v})}{d \boldsymbol{v}} \Delta \boldsymbol{v}
$$

When $\boldsymbol{K}(t)=\boldsymbol{M} \boldsymbol{T}(t)$ and $\boldsymbol{T}(t)=-[\alpha(\boldsymbol{F})]^{-1} \boldsymbol{U}_{F} \boldsymbol{\Lambda}_{q} \boldsymbol{V}_{A}$, the feedback matrix $\boldsymbol{K}(t)$ is a function of solution matrix T, which can be described by $\boldsymbol{K}(\boldsymbol{T}(t))$. The following formula is established.

$$
\boldsymbol{e}=\boldsymbol{M \varepsilon}
$$

If conditions (1)and(2) in Theorem2 are established, we can get the following relationship in the interval $t \in\left[t_{1}, \infty\right]$.

$$
\lim _{t \rightarrow \infty} \varepsilon(t)=0
$$

Then

$$
\lim _{t \rightarrow \infty} \boldsymbol{e}(t)=\lim _{t \rightarrow \infty}[\boldsymbol{w}(t)-\boldsymbol{K}(\boldsymbol{T}(t)) \Delta \boldsymbol{x}(t)]=\lim _{t \rightarrow \infty}[\boldsymbol{M z}(t)-\boldsymbol{M T}(t) \Delta \boldsymbol{x}(t)]=0
$$

We can know that the condition in Theorem 2 can meet the necessary and sufficient conditions that solution matrix $\mathrm{T}$ in Theorem 2 is nonsingular from the certificate processing, so the following formula is proved.

$$
\lim _{t \rightarrow \infty} \Delta \boldsymbol{x}(t)=\lim _{t \rightarrow \infty} \boldsymbol{T}(t)^{-1} \boldsymbol{z}(t)
$$

The certificate of Theorem 2 is over.

\section{Significance of Increment Function Observer with Variable Gain}

Completely time-invariant system is difficult to obtain in the actual physical system. The article reveals the following facts from theoretically: if matrix $\boldsymbol{T}\left(t_{1}\right)$ is existed at time $t_{1}$, which can meet 
Sylvester matrix equation $\boldsymbol{T}\left(t_{1}\right) \boldsymbol{A}\left(t_{1}\right)-\boldsymbol{F} \boldsymbol{T}\left(t_{1}\right)=\boldsymbol{G C}\left(t_{1}\right)$, there is still an additional matrix $\boldsymbol{T}\left(t_{1}\right)$ which can meet corresponding sylvester matrix equation $\boldsymbol{T}(t) \boldsymbol{A}(t)-\boldsymbol{F T}(t)=\boldsymbol{G C}(t)$, where if there are no public eigenvalue between matrix $\boldsymbol{A}(t)$ and $\boldsymbol{F}$ at any time and $\{\boldsymbol{A}(t), \boldsymbol{C}(t)\}$ is complete observability, when the parameters of matrix $\boldsymbol{A}(t)$ and matrix $\boldsymbol{C}(t)$ changes. The establishment conditions of IFO- $\boldsymbol{K} \Delta \boldsymbol{x}$ can be weakened, design of slow-time-varying systems Observer can be simplified at the same time, and the design method of IFO- $\boldsymbol{K} \Delta \boldsymbol{x}$ is similar with non-linear time-invariant controlled system. We can think that the feedback matrix $K(t)$ is a function of solution matrix $\mathrm{T}$ when the controlled object slow changes.

In the certificate processing of the sufficient conditions on Increment Function Observer with variable gain IFO-K(T(t)) $\Delta \mathrm{x}$ and the Increment full dimension State Observer with variable gain ISO- $\mathrm{T}(\mathrm{t}) \Delta \mathrm{x}$, it is different that matrix $\boldsymbol{M}$ is considered to be known and matrix $\boldsymbol{K}$ in IFO- $\boldsymbol{K} \Delta \boldsymbol{x}$ is considered to be known. The aim is to achieve the control system composite through pole placement in conventional linear system theory that matrix $\boldsymbol{K}$ is considered to be known. The matrix $\boldsymbol{A}(t), \boldsymbol{B}(t)$ and $\boldsymbol{C}(t)$ are uncertain in non-linear slowly time-varying controlled system, so there is small significance for the integrated control system assuming that the matrix $\boldsymbol{K}$ is a known constant array. The aim of designing state feedback is to improve dynamic characteristics of control system, but the steady-state indicators are guaranteed by traditional PID regulator or generalized intelligent control $^{[23,24]}$. On the one hand it is consistent with the objective reality, on the other the designing process of observer is simplified, assuming that the matrix $\boldsymbol{M}$ is a known, and feedback matrix $\boldsymbol{K}(t)$ is a function of solution matrix $\boldsymbol{T}(t)$ on Sylvester matrix equation.

\section{Summary}

A non-linear separate mode based on Hammerstein model is introduced in the paper based on transfer function description and state space description of linear system transformation. The mathematical definition and sufficient condition of Increment Function Observer with variable gain IFO-K $(T(t)) \Delta x$ and Increment full dimension State Observer with variable gain ISO- $T(t) \Delta x$ is presented, and comparing this condition with the sufficient and necessary condition of increment function observer, the useful conclusion for practical engineering can be obtained that the design of increment observer can avoid seeking the solution of Sylvester matrix equation.

In the designing process of Increment Function Observer IFO- $\boldsymbol{K} \Delta \boldsymbol{x}$, we can think that the feedback matrix $\boldsymbol{K}(t)$ is a function of solution matrix T on Sylvester matrix equation, when matrix $\boldsymbol{M}$ is known.

\section{References}

[1] Chai Tianyou, Liu Hongbo, Zhang Jing et al. Novel design method for the coordinated control system based on fuzzy reasoning and adaptive control and its application [J]. Proceedings of the CSEE , 2000, 20(4): 14-18.

[2] Luo Yi, Tian Tao, Yang Zhiyuan et al. Adaptive predictive controller with controllable gain ahead and its applications to DCS in power plants [J]. Proceedings of the CSEE, 2000, 20(10): 84-88.

[3] Huang Zuyi, Li Donghai, Jiang Xuezhi et al. Gain scheduled servo system for boiler-turbine unit[J]. Proceedings of the CSEE, 2003,23(10): 191-198.

[4] HOU Yi-wen and SHEN Jiong and LI Yi-guo. A Simulation Study On Load Modeling Of A Thermal Power Unit Based On Waveletneural Networks, Proceedings of the CSEE, 23(10): 220-224, 2003.

[5] ZHANG Chang-qing, YANG Feng, XUE Jian-zhong, et al. The Design of the $H$ Performance State Observer for the Boiler Steam Temperature Control[J]. Proceedings of the CSEE, 2006, 26(14): 109-113 (in Chinese). 
[6] HAN Zhong-Xu, QI Xiao-hong, LIU Min. A practical non-linear mathematical model of unit power plant and its application. Proceedings of the CSEE, 2006, 26(1):58-65.

[7] KONG Liang, DING Yan-jun, ZHANG Yi, et al. Application of Non-linear Dynamic Model Combined With Static Model [J]. Proceedings of the CSEE, 2008, 28(29): 93-98 (in Chinese).

[8] HAN Zhongxu. Concept of increment function observer and its application in state feedback control[J]. Proceedings of the CSEE, 2004, 24(4):210-216.

[9] HAN Zhongxu. Some design concepts of nonliear state feedback conreol system based on IFO-K $\Delta x[\mathrm{~J}]$. Proceedings of the CSEE, 2004,24(9):262-266.

[10]HAN Zhong-xu, Zhang Zhi. Application of state observer and state feedback in sub-critical boiler steam temperature control system[J]. 1999,19(11): 76-77.

[11] HAN Zhong-xu, Huang Huanpao, Zhang Zhi et al. Application of state feedback based on state observer in the coordinated control system of 300MW unit plant [J]. China Electric Power, 2004, 37(04): 60-65.

[12] HAN Zhong-xu, Wang Yuxue, Qu Yun et al. Application of state feedback based on IFO-K x in $4 \times 300 \mathrm{MW}$ genarator unit of pingliang power plant [J]. Proceedings of the CSEE, 2004, 24(12): 233-237.

[13]HAN Zhong-xu, Qi Xiaohong, Pan Gang, et al. A new design method of coordinate control system for once-through boiler and its engineering application [J]. Proceedings of the CSEE, 2005, 25(21):121-127.

[14]Han Zhongxu, Qi Xiaohong, Zhou Guang. Feed-water control scheme design of two different structure double-hearth benson once-through boilers and its application[C]. The 1st IEEE Conference on Industrial Electronics and Applications (ICIEA 2006), Singapore, 2006.

[15] Liu Min, Zhang Zhi, Wu Feijun, et al. The Design and Debuging of Main Steam Temperature Control System of 660MW Unit [J]. Modern Electric Power, 2006, 23(2): 60-63(in Chinese).

[16] HAN Zhong-xu, LV Xiu-hong, HAN Li. The robustness analysis of the state observer in the boiler super-heater temperature control[J]. Proceedings of the CSEE, 2003,23(4):177-180.

[17]Zhang Chenggan, Zhang Guoqiang. System Distinguish and Parameter Estimate, Beijing: Mechanism Industry Press, 1986.

[18] Duan Guangyin. Linear System Theory. Harbin: Harbin Industry University Press, 1996.

[19]HAN Zhong-xu, QI Xiaohong. The sufficient and necessary conditions of increment function observer [J]. Proceedings of the CSEE, 2006, 26 (23):78-82 (in Chinese).

[20]Bernard Friedlland. CONTROL SYSTEM DESIGN-An Introduction to State-Space Method[M]. New York, McGraw-Hill Book Company, 1986.

[21]Zheng Dazhong. Linear System Theory [M], Beijing: Tsinghua University Press, 2002.

[22]HUANG Youdu, DI Chenen, ZHU Shixin. Matrix Theory and Application [M], Hefei: China Institute of Science and Technology Press, 1995.

[23]HAN Zhong-xu, ZHOU Chuan-xin, ZHANG Wei, et al. New Design Method of Coordinated Control System of Supercritical Pressure Power Unit and Its Application[J]. Proceedings of the CSEE, 2009, 29(8): 75-81 ( in Chinese)

[24]HAN Zhong-xu. Designing Method of Asymptotically Tracking and Disturbance Control to Non-linear Control System [J]. Proceedings of the CSEE, 2010, 30(35): 78-87 (in Chinese) 